\title{
Creating the Context for Exploiting Linked Open Data in Multidimensional Academic Ranking
}

http://dx.doi.org/10.3991/ijes.v3i3.5023

\author{
E. Triperina ${ }^{1,2}$, C. Sgouropoulou ${ }^{1}$, I. Xydas ${ }^{1}$, O. Terraz ${ }^{2}$ and G. Miaoulis ${ }^{1,2}$ \\ ${ }^{1}$ Technological Educational Institute of Athens, Egaleo, Greece \\ ${ }^{2}$ University of Limoges, Limoges, France
}

\begin{abstract}
Academia is a complex socio-technical system with multiple aspects and constituents that involve various stakeholders. In order to address stakeholders' needs and to assist the institutional accountability, this complexity should be considered during the development of academic services. We have designed a dynamic multidimensional ranking approach, easily modifiable to address user requirements, so as to assess and compare the university performance with a clear view to the support of effective institutional strategic planning and policy making. Our approach comprises the following components: the AcademIS ontology to model the academic domain and its multiple dimensions, the AcademIS Information System to manage and display the academic information, published in Linked Open Data format and the visual-aided Multiple Criteria Decision Making component, to evaluate and rank the performance of the academic units. The data are aggregated from several sources, in different formats, LODified by our system, and presented to the user by the interface to ultimately assist the decision making process.
\end{abstract}

Index Terms-Academic Linked Open Data (LOD), Ontology, Multidimensional ranking, Visual analytics..

\section{INTRODUCTION}

Nowadays, information about the Higher Education Institutions (HEIs) and their performance in various fields has become widely available to the public. Based on this information, insights can be derived about the degree in which a higher education institution fulfills its tasks and reaches its goals. In order for a HEI to show in a clear and understandable manner its performance, all the information must be available to the parties involved. The stakeholders of academic institutions involve: faculty, current and future students, policy makers and decision makers of Higher Education, potential industrial collaborators and society at large. In order to have informed insights, each category of stakeholders needs to access different data:

- an academic wants to access information related to the research conducted, the courses and the faculty,

- a future student seeks information related to the curriculum, the faculty and the quality of the offered services,

- a potential collaborator needs to be informed about the research, the patents, the services and the products developed by the institutions,

- while a policy maker may need all the above mentioned information.
As a direct consequence, the related information about the involved domains should be captured. This information, not only has to be accessible - thus open - to the related users, but it should also be reusable - thus stored using a reusable data format. In the majority of HEIs this information is scattered across numerous institutional databases. By aggregating all the data in one information system, which does not substitute the already existing systems, the management and the exploitation of academic information by all the related stakeholders becomes much more efficient and easier. When this information is supposed to be used in the context of evaluation, it is of vital importance to ensure its transparency by promoting the validity and the reproducibility of the applied processes and the achieved results.

The motivation for developing the AcademIS ontology and the homonymous information system was the need to accumulate the whole academic information in a single information system. Further motivation stems from the necessity to facilitate a transparent, reproducible and multidimensional academic ranking method for the Higher Education setting using visual analytics and Linked Open Data (LOD). The proposed approach examines how academic information can be processed and visualized to efficiently respond to stakeholders questions and seeks the appropriate criteria to evaluate the academia. Based on these criteria an ontology is created for the domain of Higher Education. The ontology is used as the backbone of the semantic web information system and its visualaided Multiple Criteria Decision Making (MCDM) module. Its role is crucial, since it guarantees the existence of the required multidimensional fields and also soothes the transition between different application domains. The information system hosts and presents the linked open academic data and its multiple dimensions that correspond to activities and cooperations related to education, research and collaboration with the Industry. AcademIS aims to evaluate the universities, to aid the decision making process, and to dispose the data in a visual way that is comprehensible for all the involved parties. It provides a mechanism for multiple purposes that is qualitative and adaptive.

In the first section, there is the introduction, while the second section provides the literature review. Section 3 reveals the methodology and the section 4 offers a description of the distinct components - the results - of our approach, which include the multidimensional ranking method and the underlying algorithm, the academic domain model, the AcademIS information system, and the LOD in our approach. Section 5 outlines the conclusion. 


\section{LITERATURE REVIEW}

Our approach is focused on developing an information system with decision making capabilities that utilizes the semantic web technologies. It is based on an academic domain model, which incorporates the necessary multidimensional information and also provides the facilities for the LOD generation and the multiple criteria ranking. In the following section, the background information for all the key concepts of our approach is discussed. To be more specific, the most important HEI ranking methods and their main characteristics are described and the required background for the visualaided MCDM is outlined. The existing academic domain models and their scope are also presented. Subsequently, the importance of Linked Open Data and the semantic organization of the information for the HEI setting are referred.

\section{A. Multidimensional ranking of HEIs}

Rankings and more specifically global rankings are a popular method to compare the universities worldwide. Nevertheless, rankings are prone to problems. First and foremost, their majority is keen to rank higher the research universities and to neglect the variety of universities' purposes [1]. Most of the rankings take into account only research indicators, while other approaches, which incorporate more academic indicators, tend to affect the results to the benefit of research, by assessing greater weights to the indicators related to research [1]. Moreover, usually rankings are occupied with the whole institution and they do not assess each academic department separately. However, the sector, the purpose and the performance differ in each department of an academic institution. Furthermore, since the ranking approaches reflect the opinions of their creators on the importance of each university aspect, the outputs can be considered as biased. The end user should be able to modify the criteria and their indicators. Several rankings aim to evaluate a selected pool of universities, while other approaches attempt to rank all the universities at once. However, by ranking a group of a-priori characterized good universities, the results become prejudiced. On the other hand, attempting to rank all the universities at once, raises severe difficulties associated to the accumulation of the data [2]. An alternative approach is to personalize the outputs by applying the ranking to the universities of interest of the user. Rankings also incline to present only the products of the process and do not provide direct or open access to their data, which may cause problems, related to their reproducibility and their transparency [3]. Besides the criteria that are related to the education, research, the cooperation with the industry, the regional and the international aspects - the social facets of academia should be also addressed. The need for capturing academic social responsibility has been also pinpointed in [4].

In the following paragraph, the ranking approaches and their characteristics will be outlined. Among the existing methods, the most important are the U-Multirank approach [1], the Times Higher Education (THE) [2], the Shanghai ranking [5], the Leiden ranking [6], the CHE ranking [7], and the Taiwanese college navigator method [8]. U-Multirank is an EU funded project that proposes a multidimensional ranking approach, considering the following dimensions of the academic endeavors: teaching
- learning, research, knowledge transfer, international orientation and national engagement. It aims to evaluate and rank all the universities and colleges. The Times Higher Education (THE) ranking method employs thirteen separate performance indicators categorized in the following groups teaching, knowledge transfer, international outlook, research and industry incomeinnovation. In THE ranking, the research related indicators have a greater weight than the other indicators. THE rankings are applied to a specific set of universities derived from the list of world's leading research universities by Thomson Reuters. The Shanghai ranking includes six different criteria, two related to the awards won, which are the alumni (the amount of Nobel prizes of alumni), and the award (the number of Nobel prizes of faculty) and four more indicators that reflect the quality of research, namely the $\mathrm{HiCi}$ (the number of highly cited researchers), the NCS (the number of articles published in journals Nature and Science), the PUB (the number of articles indexed in the science citation and the social science citation index) and the PCP (a weighted average of the scores obtained in the five prior categories divided by the amount of the academic excellence) [9]. All the indicators of the Shanghai rankings are research related indicators. The use of the weighted average of the already accounted indicators is considered to distort its outputs. Leiden ranking is a global university ranking based on bibliometric data and includes three citation index indicators the mean citation score (MCS), the mean normalized citation score (MNCS) and the proportion of top $10 \%$ publications (PPtop10\%), as well as 4 scientific collaboration indicators, which are the following: the proportion of collaborative publications (PPcollab), the proportion of international collaborative publications (PPint collab), the mean geographical collaboration distance (MGCD) and the proportion long distance collaborative publications $(\mathrm{PP}>1000 \mathrm{~km})$ [6]. All the indicators of the Leiden ranking are also research-focused. The CHE (Centre for Higher Education Development) university ranking has the following components: student body, student outcomes, international orientation, teaching and learning, infrastructure, research, labor market, study location and university and the overall assessment by students and professors [7]. It evaluates only departments and its main aims are to assist student choice and aid the institutions to find their strengths and their weakness. The Higher Education Evaluation and Accreditation Council of Taiwan (HEEACT) 's College Navigator in Taiwan is another ranking approach that has the following criteria: academic survey, library acquisitions, student quality, faculty resources, research grant, research output, teaching quality, learning output and international outlook [8]. It is a personalizable ranking approach with predefined criteria and indicators that offers the possibility to the users to set their preferences related to location, size, type and discipline of the academic institution.

Some considerations must be taken into account when ranking universities. First and foremost, rankings have better results if they are applied in groups of similar institutions, which means that a classification should be applied upon the universities to be ranked. Apart from research, all the facets of academia must be represented as well. University rankings should be balanced regarding the appointed dimensions, their individual criteria and the respective weights. Moreover, ranking approaches should 
be flexible concerning the scope (university-wise, department-wise), the subjects (the specific universities or departments) and the characteristics (dimensions, criteria and weights) of the assessment. The method, the data and the ranking findings ought to be open and accessible.

\section{B. Visual-aided multiple criteria decision making}

The MCDM algorithm and the visual analytics are the components of the visual-aided MCDM. In the following paragraphs, these topics will be described. Multiple Criteria Decision Support Systems (MCDSS) are Decision Support Systems (DSS) that aid the structure and the solution of a Multiple Criteria Decision Making (MCDM) problem [10]. Ranking constitutes one of the problems that MCDM addresses. Among the most significant MCDM approaches are the outranking method, the multiattribute utility theory, the Analytical Hierarchy Process (AHP). The benefit of the ELECTRE compared to the other approaches is the absence of compensation between the criteria. ELECTRE methods cover choice, sorting and ranking problematic. The problem of ranking is answered by the following ELECTRE algorithms: ELECTRE II, ELECTRE III and ELECTRE IV [11]. The ELECTRE III ameliorates ELECTRE II and can deal with imprecise and ambiguous data [11], while the key difference between the ELECTRE III and IV is the use of relative importance indices in the former [12].

Data visualizations have been utilized on various fields, due to their many advantages and MCDM should not constitute an exception. However, there is a limited amount of approaches that combine MCDM with visual analytics, especially on the ranking problematic. Based on the interactive visualizations, meaningful insights for the data and their connections can be provided to the user [13]. Through them the users are eased to detect the relations between the data and the patterns that are present via their perceptual abilities [13, 14]. Although the human perceptual abilities are narrow when analysing raw data, the visual system affords massive parallel processing capabilities [14].

The added value of visualizations is that they enable the deeper comprehension of the perplexed multidimensional information, revealing relations that otherwise will not be apparent [14]. More specifically, the domain of visual analytics assists analytical reasoning by exploiting the human perception capabilities about complex data [15]. As a result, by merging the visual analytics component with the MCDM, the decision making process is simplified and the stakeholders are considerably aided. A visualization type that is ideal for the representation of multidimensional datasets is the parallel coordinates representation [16].

\section{Modeling academic activity}

The academia encompasses many different activities and functionalities, ranging from education and research to administrative duties and cooperation with the industry. There are numerous modeling approaches for the academic activity. Nevertheless, each approach focuses on a different aspect of the academia, thus each domain model has a different scope.

The most prominent academic modeling approaches can be categorized in research, educational and academic ontologies. The latter category includes research and education, as well as other concepts of academia. The research ontologies are CASRAI and CERIF, the educational ontologies are OMNIBUS, HERO, Ontoural, Ontology of Instructional Items and AIISO, while VIVO, HERO and Univ-Bench can be classified as academic ontologies. Research ontologies focus on the researchers, the research products and procedures. CASRAI (The Consortia Advancing Standards in Research Administration Information) standards refer to the terminology of the semantics and the structure of research information [17], the life-cycle of research and the research impact [18]. CERIF (Common European Research Information Format) corresponds to a canonical reference data model for data and metadata about research objects and their relationships [19, 20]. Different concepts of education are represented by the educational modeling approaches. To elaborate, the OMNIBUS ontology provides a detailed modeling of learning instruction, instructional design and the concrete phenomena of education [21]. The Ontology of Instructional Items catalogs the "instructional semantics" of learning resources terminology [22], while the Ontoural, facilitates ontology-based learning environments by mapping the main actors and contexts of the learning process [23]. The AIISO ontology (Academic Institution Internal Structure Ontology) is more focused on the internal organization of academic institutions [24]. The ontologies that model research, education and the other aspects of academia are usually more concentrated on one of these domains. The VIVO ontology is used as a basis for the homonymous open source semantic web application. The VIVO-ISF (Integrated Semantic Framework) ontology [25] includes research concepts and relationships, as well as several basic educational concepts, such as the actors involved in education (professors), and the educational products (course, workshop, etc.). The HERO (Higher Education Reference Ontology) models the characteristics of university domain [26], while the Univ-Bench represents the university domain and facilitates the evaluation of Semantic Web repositories [27].

Even though there are modeling approaches that encapsulate the academic activity, they are failing to capture all the dimensions of HEIs and their interconnections. They do not consider academia as an area with multiple domains, and as a result they do not reflect its multiple aspects.

\section{Semantic organization of the data}

Currently, the growth and the availability of data are exponential. Linked Data is a manifestation of this fact. Linked Data offers structure to data and connections through the datasets, whereas Linked Open Data provides also the benefits of open access. The Linked Open Data cloud is composed from all the available interconnected datasets of Linked Open Data and has been growing continuously [28], since its launch in 2007. Until October 2014, there have been 570 linked datasets, connected by 2909 linksets [29]. The LOD cloud includes information derived from different fields [30], such as media, geographic, government, publications, cross-domain, life sciences and user-generated content.

Many studies have proven the importance of transforming relational data into Linked Data format [31, $32,33]$. Ontologies ease the transition between relational databases and Linked Data, since they express the relationships among concepts within a particular domain 
PAPER

CREATING THE CONTEXT FOR EXPLOITING LINKED OPEN DATA In MultidimENSIONAL ACADEMIC RANKING

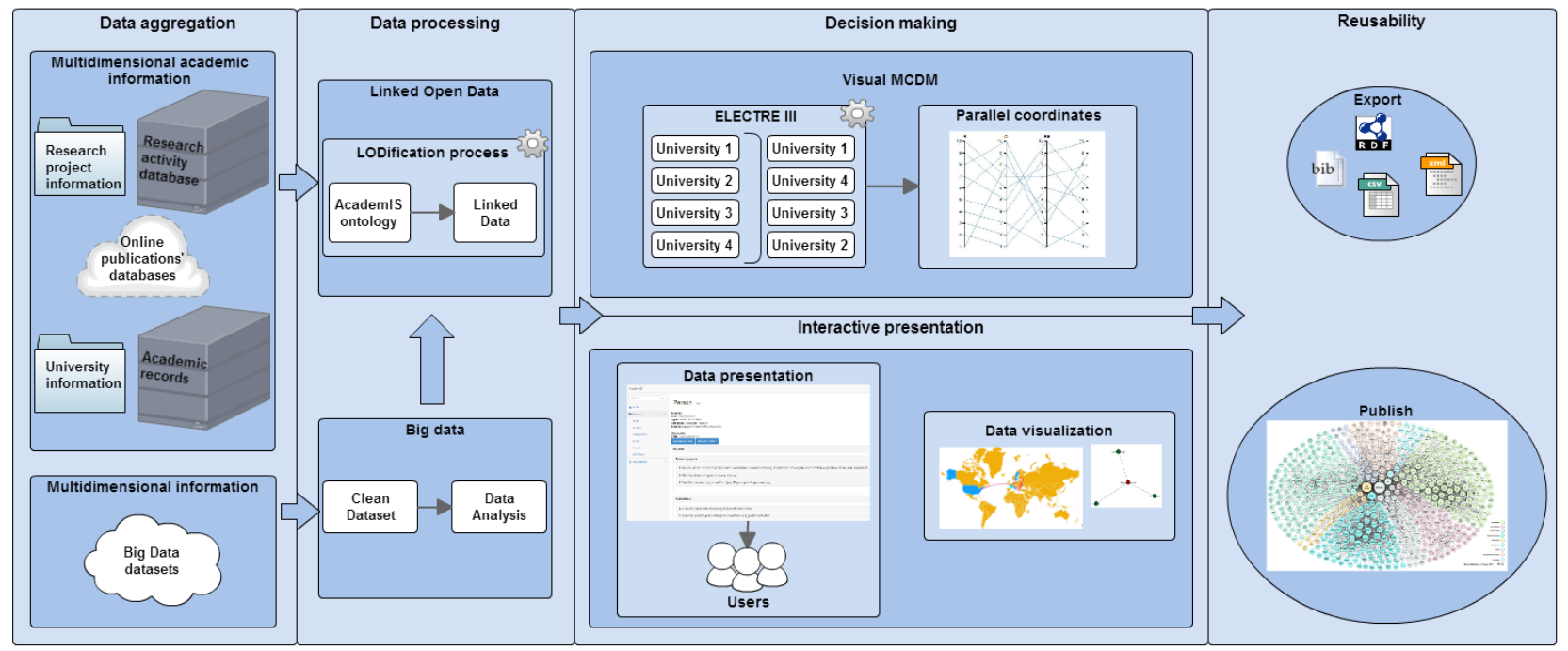

Figure 1. AcademIS architecture (Adapted from [36])

and provide the structure of Linked Data [34]. Reproducibility [35], shareability, extensibility, reusability and the fact that applications can deal with them directly [28] are some of the benefits of Linked Data.

As mentioned before, it is essential for the rankings to provide the institutional data in a reusable and digital format that can be understood by both people and machines, so as to make the ranking procedure more transparent. Linked Open Data ensures these requirements. Moreover LOD assure the validity and the reproducibility of the ranking process and its outputs.

\section{Methodology}

Our university ranking method is supported by the development of a modeling approach for the academic domain. The domain model captures all the facets of academia and the criteria that measure the performance of these facets. Finding the most important criteria for each academic facet, deciding on the gravity that each criterion should have and integrating them into the domain model are essential for this process. The criteria and their weights are defined in the ontology and aligned to the respective fields of the MCDM method. In that way, the necessary information for the MCDM is derived from the knowledge base.

The institutional data are aggregated to the system and structured with the aid of the academic domain model. The data are retrieved from various valid sources on the web, such as research information systems, like Scopus, along with data from institutional databases, university records and institutional research management systems, such as I.RE.MA. [37]. The format of the information provided by each source ranges from relational databases, to Linked Data and to other reusable data formats, such as comma-separated values (csv), BibTeX, JavaScript Object Notation (json), etc. Once the data have been accumulated to the system, they are processed and unified by the LODification process, which is based on the AcademIS ontology. Thereafter the data are presented to the user by the interface of AcademIS information system. AcademIS interface presents the stored data, in profiles of academics, courses, research, publications and projects, while another feature of the system allows the user to compare the departments of an institution with one another, or with the departments of other institutions, as well as the whole institution to other institutions. The information is also presented with interactive visualizations that are implemented with JavaScript. The AcademIS information system is implemented in Java using the Jena Framework that supports the semantic features needed in our application. The input needed for our system is multidimensional data, while the outputs of the system are the data presented by the interface and the visualized results of the DS mechanism. First and foremost, due to the utilization of ontologies the data hosted in the system have a common format, which is also required by the ontology-based DSS component. With the use of the ontology, we ensure that i) the required dimensions for the ranking method exist, ii) the data are unified and iii) have a compatible form with the DSS component. Then the MCDM ranking algorithm is applied in the data and the results are conveyed to the visual analytics module, which facilitates the presentation of the ranking results and assists the decision making of the user.

In terms of data, the process is also applicable in Big Data with several modifications in the data processing stage. However, the further analysis of these facets of our system is out of scope of this paper. In terms of the domain, the methodology can be also adjusted to other fields, provided the multidimensional character of the data and the existence of multiple criteria in that domain. The procedure that should be followed is the following: first, an appropriate ontology for the domain must be specified, the multidimensional criteria should be paired to the related fields in the specified domain and the appropriate modifications should be carried out to the interface. After these modifications, the system is ready for use.

\section{RESUlTS}

The AcademIS Information System manages and visualizes the institutional data and also hosts the multidimensional ranking of HEIs. The components of the proposed solution are presented in this section, including the multidimensional aspects of AcademIS, the MCDM ranking algorithm and the academic ontology. Thorough descriptions of the information system, as well as the use of LOD in our approach are also provided in this section.

The novelty of our approach is the introduction of visual analytics in the MCDM process and the support of this process by an ontology. Another contribution is the 
development of rules in a system based on VIVO. Apart from the rules, elaborate concepts and relations have been added to the ontology to cover quality and accountability aspects of HEIs, as well as the multidimensionality of the academic domain. Finally, the availability of the multidimensional ranking information in LOD is another novelty, since the majority of ranking systems do not offer the data in which they have based their ranking and even those rankings that have an open data policy, they do not provide their data in such a format.

\section{A. Academic multidimensional ranking}

Evidently, all universities do not share common goals and objectives. There are universities that focus on research, other universities set as priority their mission on education, while others promote another aspect of academia. Each university has many goals and missions. So, the use of a unique dimension is not advisable in university ranking, since they have multiple undertakings and responsibilities. In case of academic evaluation and ranking, a multidimensional approach should be utilized to assess all the subdomains of academia. However, as stated before, since universities' goals vary, it is essential to avoid a ranking approach with fixed dimensions. Instead, a more personalizable ranking should be adopted, that allows the definition and the modification of ranking characteristics depending on the stakeholders' needs.

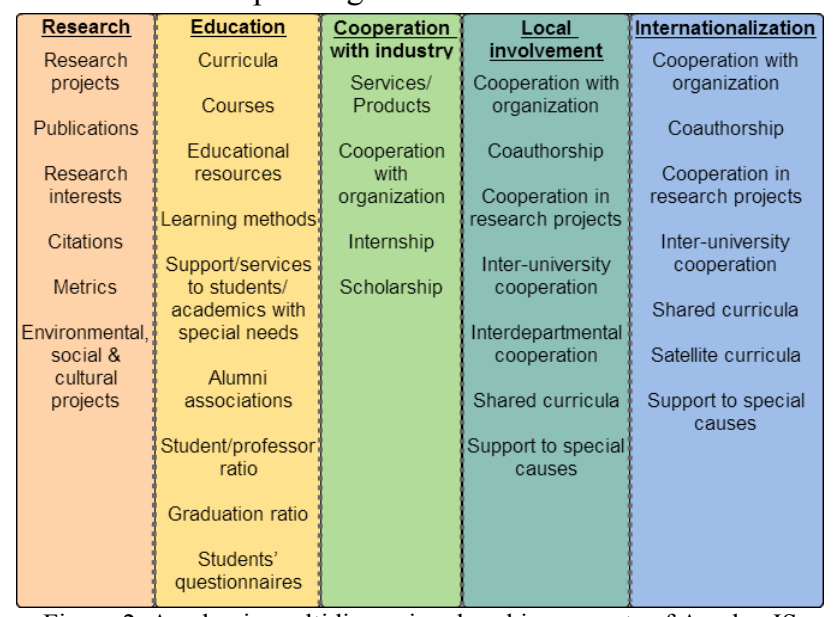

Figure 2. Academic multidimensional ranking aspects of AcademIS

Each of the dimensions has several characteristics. These characteristics will be referred to as criteria. In each criterion a weight is assigned that delineates its relevance and gravity in its dimension. In the development stage of this approach, the available ranking approaches have been taken into account, with special focus on the U-Multirank method. The dimensions considered in our approach are Research, Education, Cooperation with industry, Local involvement and Internationalization. The knowledge transfer dimension of U-Multirank is not considered as a separate dimension in our approach, but some of its criteria have been assimilated in the other dimensions. The criteria in each dimension are focused on the following themes: the activities, the collaborations, the evaluation, the social responsibility and the impact of the academic institution. Our approach introduces several criteria for measuring the academic social responsibility (such as the participation in environmental, social and cultural research projects, the support to students and academics with special needs, the alumni associations and the support to special causes) that do not exist in another ranking approach. In Figure 2, the dimensions of academic multidimensional ranking, as well as their criteria are presented. The ranking is implemented through the visualaided MCDM method that is presented in the next section.

\section{B. Visual-aided multiple criteria decision making}

In this part, we describe the visual multiple criteria decision making component of our system, which combines a MCDM approach with visual analytics to assist and enhance the decision making process of the involved parties.

In our approach, a technique from the ELECTRE family, which is suitable for our ranking purposes, is selected and modified. To elaborate, we have implemented a variation of ELECTRE III algorithm that takes as input multidimensional data structured as Linked Open Data. The outputs of the ontology-based ELECTRE III supply our visual analytics component, which is a parallel coordinates visualization. Parallel coordinates are used for the visualization of multidimensional data of moderately sized datasets and our dataset meet these criteria. To further exploit the benefits of the above mentioned visual representation, we apply statistical coloring of the represented data, based on the value of the records of a specific dimension, namely the education. Through the parallel coordinates visualization the data become traceable. The final result of this component is the visualized data represented in the axes of parallel coordinates.

By combining ELECTRE III algorithm with visual analytics, the information can be processed faster and easier. As a direct consequence, the decision making process is getting more effective and efficient. The required information for the ranking, including the dimensions, the criteria and their weights are captured through the AcademIS ontology.

\section{The academic domain model}

The AcademIS [38] multidimensional academic domain model provides the means to structure the information needed for the multidimensional ranking of universities. The upper ontologies of AcademIS are: Bibontology, Dublin Core elements, Dublin Core terms, Event Ontology, FOAF, geopolitical.owl, Provenance support Research Resources (eagle-i), Scientific Research, SKOS, Vitro Public Ontology and VIVO core. AcademIS (Figure 3) covers core academic concepts, processes and multidimensional aspects. It also includes important terms relevant to the complex relationships formed among the various dimensions of academia, quality assurance metrics and social accountability information.

The domain of our ontology is the academia, whereas its main components are i) its actors, which include professors, staff, collaborators, researchers, graduate and undergraduate students, ii) the academic activities and iii) the collaborations. The purpose of building the AcademIS ontology is to model the whole academic domain and to support the evaluation and the ranking of the Higher Education. An excerpt of the competency questions of the AcademIS ontology, one for each academic dimension, is presented in the Table 1. The ontology is used as the basis of the AcademIS Information System, where it is utilized as a structure prototype for the institutional LOD publishing and aids the MCDM by aligning the concepts 


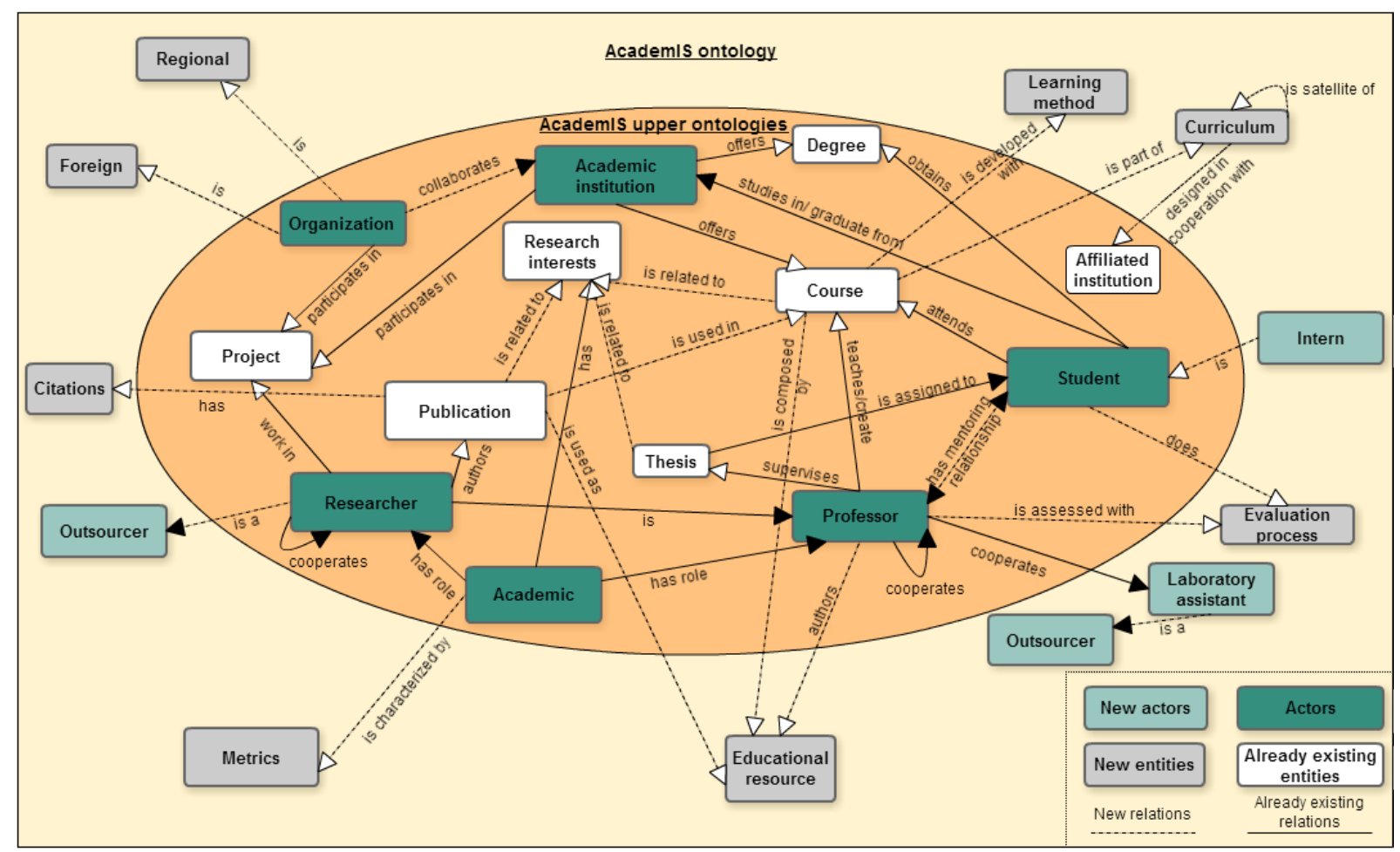

Figure 3. AcademIS ontology in relation with its foundational ontology VIVO

of the academic domain to the criteria of the decision making process. It examines the aspects of academic institutions, including research (projects, publications, books, patents), education (courses, workshops, thesis), regional and international relationships (shared curricula, joint publications, collaborations in research projects), as well as relationships with industry (scholarships, internships), concentrated on the performance indicators. The intended end users are the stakeholders of academic institutions, including current and future students, possible collaborators, policy makers of academic institutions, and the faculty. Its main indented use is the facilitation of academic quality management and ranking.

TABLE I.

COMPETENCY QUESTIONS OF THE ACADEMIS ONTOLOGY

\begin{tabular}{|l|l|}
\hline An excerpt of the competency questions for AcademIS ontology: \\
\hline Research & $\begin{array}{l}\text { i) Which characteristics of research } \\
\text { publications are useful for defining the quality } \\
\text { of research? }\end{array}$ \\
\hline Education & $\begin{array}{l}\text { ii) What types of courses are there in the } \\
\text { university? }\end{array}$ \\
\hline $\begin{array}{l}\text { Cooperation } \\
\text { with industry }\end{array}$ & $\begin{array}{l}\text { iii) Which characteristics must an organization } \\
\text { have in order to host interns from the academic } \\
\text { institution? }\end{array}$ \\
\hline Local involvement & $\begin{array}{l}\text { iv) What are the prerequisites for an } \\
\text { organization to cooperate with an academic } \\
\text { institution? }\end{array}$ \\
\hline Internationalization & $\begin{array}{l}\text { v) What is the process for an academic } \\
\text { institution to cooperate with another institution } \\
\text { to create a shared curriculum? }\end{array}$ \\
\hline
\end{tabular}

To better present the AcademIS ontology and the underlying concepts and relationships, the ontology expressed in Description Logic (DL) and the meaning of each sentence are displayed in the Table 2. Fragments of the TBox, RBox and ABox are presented, which respectively contain sentences that describe concept hierarchies, rules and instances. For the sake of brevity, we include only an excerpt of our ontology in DL.
TABLE II.

TBOX, RBOX AND ABOX OF ACADEMIS ONTOLOGY IN DL

\begin{tabular}{|c|c|}
\hline \multicolumn{2}{|l|}{ TBox } \\
\hline DL & Meaning \\
\hline ProfessorபResearcherᄃAcademic & $\begin{array}{l}\text { Every professor or researcher is } \\
\text { an academic. }\end{array}$ \\
\hline Professor $\sqsubseteq \forall$ teachesOf.Course & A professor teaches a course. \\
\hline $\begin{array}{l}\text { UndergraduateStudent } \sqcup \text { Graduate } \\
\text { StudentㄷSStudent }\end{array}$ & $\begin{array}{l}\text { Every undergraduate or graduate } \\
\text { student is a student. }\end{array}$ \\
\hline $\begin{array}{l}\text { ResearchOrganization } \subseteq \text { Organizat } \\
\text { ion }\end{array}$ & $\begin{array}{l}\text { Every research organization is } \\
\text { an organization. }\end{array}$ \\
\hline 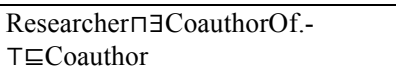 & $\begin{array}{l}\text { A researcher, who has somebody } \\
\text { as coauthor of, is a coauthor. }\end{array}$ \\
\hline $\begin{array}{l}\text { Professor } \square \exists \text { CollaboratorOf.- } \\
\text { ТᄃCollaborator }\end{array}$ & $\begin{array}{l}\text { A professor, who has a } \\
\text { collaborator of, is a collaborator. }\end{array}$ \\
\hline $\begin{array}{l}\text { Organization } \sqcap \exists \text { CollaborationWit } \\
\text { h.AcademicDepartment } \\
\text { Affiliated Organization }\end{array}$ & $\begin{array}{l}\text { An organization that has } \\
\text { collaboration with academic } \\
\text { department is an affiliated } \\
\text { organization. }\end{array}$ \\
\hline $\begin{array}{l}\text { UndergraduateStudentㄷ} \exists \text { takesInt } \\
\text { ernshipIn.RegionalOrganization }\end{array}$ & $\begin{array}{l}\text { An undergraduate student takes } \\
\text { internship in } \\
\text { organization. }\end{array}$ \\
\hline $\begin{array}{l}\text { UndergraduateStudent } \subseteq \exists \text { registere } \\
\text { dIn.EducationalProgram }\end{array}$ & $\begin{array}{l}\text { An undergraduate } \\
\text { registered in } \\
\text { program. }\end{array}$ \\
\hline $\begin{array}{l}\text { Evaluation } \subseteq \forall \text { cinductedBy.Studen } \\
\text { tபEvaluator }\end{array}$ & $\begin{array}{l}\text { Every evaluation is conducted } \\
\text { by a student or an evaluator. }\end{array}$ \\
\hline \multicolumn{2}{|l|}{ RBox } \\
\hline DL & Meaning \\
\hline teacherOf $\equiv$ stu & $\begin{array}{l}\text { teacher of and student of are } \\
\text { inverse roles }\end{array}$ \\
\hline takeInternship $\equiv$ offerInternship- & $\begin{array}{l}\text { take internship and offer } \\
\text { internship are inverse roles }\end{array}$ \\
\hline authorOf드eatc & author of is a subrole of creator of \\
\hline \multicolumn{2}{|l|}{ ABox } \\
\hline DL & Meaning \\
\hline $\begin{array}{l}\text { \{Distributed } \quad \text { Programming, } \\
\text { Operating Systems } I\} \subseteq \text { Course }\end{array}$ & $\begin{array}{l}\text { Distributed Programming and } \\
\text { Operating Systems I are courses }\end{array}$ \\
\hline$\{\mathrm{GM}, \mathrm{CS}, \mathrm{IX}\} \subseteq$ Academics & $\mathrm{CS}, \mathrm{GM}$, and IX are academics \\
\hline $\begin{array}{l}\text { \{TEI of Athens, University of } \\
\text { Limoges }\} \subseteq \text { Academic Institutions }\end{array}$ & $\begin{array}{l}\text { TEI of Athens and University of } \\
\text { Limoges are academic } \\
\text { institutions }\end{array}$ \\
\hline
\end{tabular}


Our contributions regarding the academic domain model are the following: the concepts and the relations regarding the collaborations between academics, the multidimensional aspects of academic institutions, the evaluation of the Academia and the academic social responsibility. Furthermore, our approach introduces rules to the academic ontology to assist the evaluation process. It must be mentioned that this system is the first VIVObased system that uses Semantic Web rules to trigger the creation of information essential to the ranking method. Specifically, several criteria of the MCDM are generated through the inference mechanism. The AcademIS domain model provides the concepts, the relationships and the rules that govern the data facilitated in the corresponding information system.

\section{The AcademIS Information System}

In this section the basis of AcademIS will be discussed, the components of the information system and the scenarios of its usage will be presented. The foundation of our system lies to I.RE.MA. [37] a research management system. I.RE.MA. uses the research related information of an institution to build informative visualizations from which meaningful insights on the faculty's research performance can be derived. AcademIS builds on I.RE.MA. and provides additional features in terms of data, user interface, technologies, components and offered services.

First and foremost, in terms of data management, the AcademIS introduces the following concepts in relation to I.RE.MA.. It utilizes relational databases and reusable data formats and LODifies them with the corresponding method. As a result, the data hosted in the AcademIS application are unified and available as Linked Open Data. Regarding the data itself, it not only includes the research information of an institution, but also incorporates educational information, data about the cooperation with the industry and other domains. The information that AcademIS hosts is multidimensional. As far as the user interface is concerned, AcademIS offers a way to manage and display the information available in I.RE.MA. in form of profiles. A unique profile is available for each academic concept, which contains all the related information it. It also employs semantic web technologies in order to take advantage of the widely available datasets and increase the reusability and transparency of the academic data. Several components have been developed, which broaden the range of functionalities offered by the Institutional Research Management system. I.RE.MA. offers the possibility to produce insights about the performance of the individuals, whereas AcademIS focuses more on the performance of an academic unit. In terms of the information system, AcademIS support the management and presentation of academic related information in LOD format, while it also utilizes this information to derive useful insights by implementing a MCDM ranking method on them and visualizing the results in order to allow the user to make the final selection.

AcademIS is a semantic web application that is based on the corresponding ontology to create academic LOD that provides the following services: the transformation of data to LOD, the management of academic information, the academic ranking and evaluation. These services are offered by the LODification mechanism and the AcademIS interface. Each of these components, along with usage scenarios of the system, will be further described in this section.

\section{1) Data in AcademIS}

LODification of the data allows the creation of an unambiguous, unanimous, easier manageable and shareable set of data. This process enables the input of the aggregated data from disparate data sources, such as relational databases and web services. Based on the AcademIS ontology the LODification method converts the data in RDF format and creates the Linked Data records. The data imported into the system by the interface are undergone the same process. All the acquired information is available in the knowledge base, where users can retrieve and access it through the interface and the interactive visualizations. They can also export it and reuse it in a LOD compliant system.

The LODification process is influenced by the approach of [39] and consists of the following phases: i) data are aggregated from the various sources, or input to the system via the AcademIS interface, ii) information is mapped to the correct concepts of the ontology, so the domain translation takes place, iii) the records are transformed in Linked Data format, iv) the Linked Data records are then ready for storage in the knowledge base and available from the user interface.

It must be mentioned that there is not another MCDM or multidimensional ranking that employs LOD in its method. However, AcademIS supports the multidimensional institutional LOD records throughout their lifecycle, including their creation, publication, management and curation. It also fosters the multiple dimensions evident in the academia.

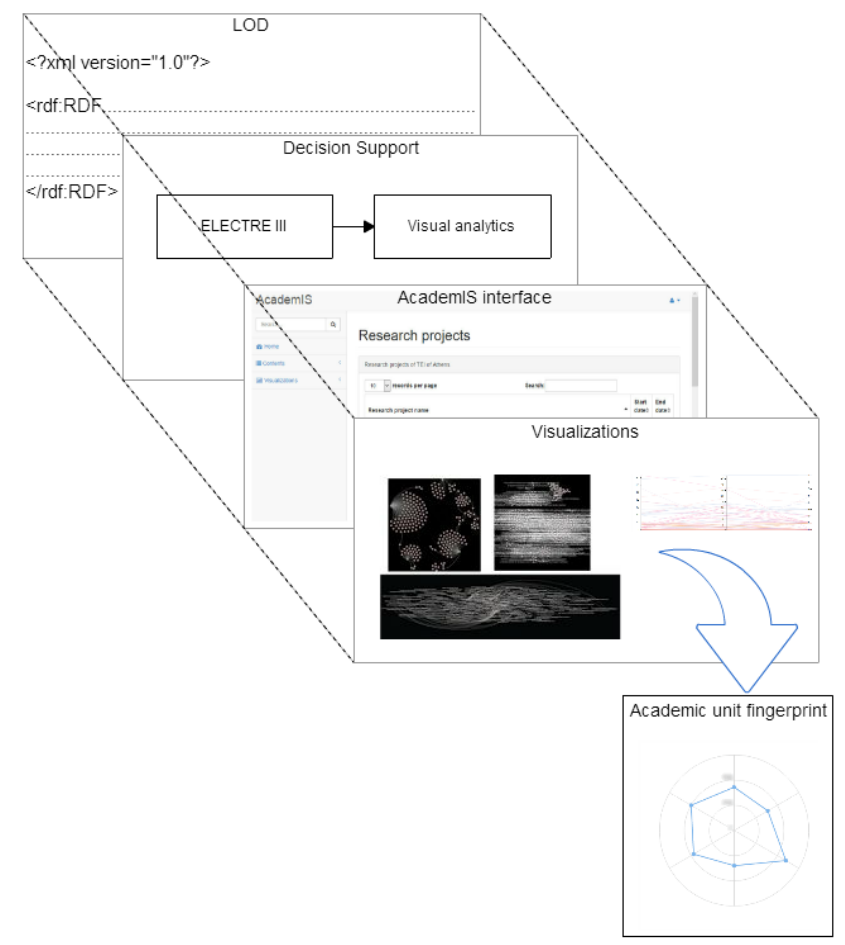

Figure 4. The layers of AcademIS Information System

2) The AcademIS interface 
AcademIS interface hosts profiles of academics, the conducted activities and the offered services, as well as interactive visualizations of the data. The interface provides access to additional information about academics, academic activities that happen in a HEI, for instance courses, workshops, publications, projects, patents, as well as collaboration with regional or foreign organizations. Hence, it promotes the multidimensionality of academic institutions and accumulates the related information, while at the same time it offers an interactive way to manage it. Thus, except from constituting a ranking approach for the universities and their departments, it also provides a management interface of the university's information and the individual data of the academics.

\section{3) AcademIS visualizations}

The information is displayed in textual format and also with the use of visualizations. The visualizations that are provided by our system are matched in the following categories: i) visualizations of the relations in academic activities among individuals, such as the collaboration graph provided by I.RE.MA. (Figure 5), ii) spatiotemporal visualizations that disclose the cooperation patterns formed in the academic setting with regard to time and space and iii) visualizations of the multidimensional ranking results. In the latter category, two types of visualizations have been developed, namely the multidimensional comparative ranking and the academic unit fingerprint.

Relationships visualizations capture the associations between the faculty of the academic institution in the academic setting, the connections between the academic units and the organizations. Spatiotemporal visualizations reveal the evolution of academic activity in relation with the time and capture the relationships between the institutions and organizations based on their spatial information. In the category of multidimensional visualizations, the comparative performance of two or more institutions based on predefined or user defined criteria is presented. It shows the institution's performance compared to the performance of other academic units, based on a multidimensional analysis. The multidimensional method creates a context in which each institution can be assessed by associating it to other institutions. By collating academic units in more than one dimension, the stakeholders can descry in which dimension each institution outmatches others and where it falls behind, resulting in a more detailed and concrete approach. The multidimensional comparative ranking produces an overview of the performance of academic institutions, allows users to correlate them and to make deductions which assist the decision making process. The academic unit fingerprint visualization compares the selected academic unit with prototype profiles, which have a specific set of indicators designated to them. These profiles can be either predefined or user defined. The academic unit fingerprint provides insights on how a specific institution performs in a single dimension, bearing in mind the individual criteria of this dimension. The different ranking patterns of the academic unit fingerprint can host the needs of the Industry, the Academia and other sectors. For instance, if the user wants to inspect the performance of an institution in the dimension of education, the criteria that are linked with this dimension should be presented. In the above described scenario, the ratio of students-professors, the percentage of graduates, the offered courses and learning activities, as well as other criteria that clarify the quality of education in an institution are taken into account.

AcademIS considers the diversity of the intended users and includes multiple ways of displaying the data for different purposes to ease their comprehension. Furthermore, it employs different visualization approaches based on a number of facets, such as the type of the data to be presented, the amount of data and the relationships between the data.
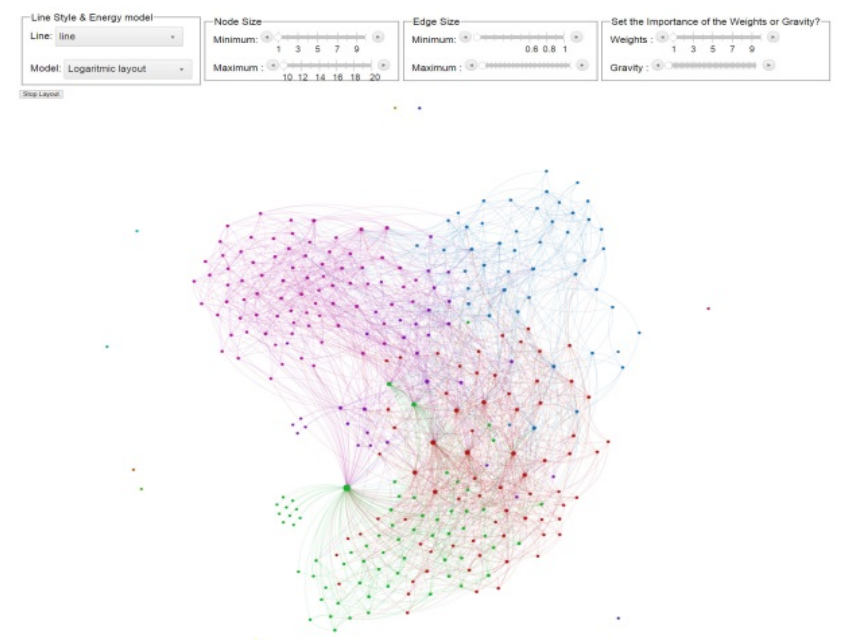

Figure 5. Collaboration graph

\section{4) AcademIS use cases}

In this section, we demonstrate how the AcademIS system can be employed so as to satisfy several use cases. The use cases (Table 3) present the different contexts where our system can be utilized. They outline the components of the system and the sequence in which they are used. AcademIS intends to satisfy the needs of multiple user groups, including academics, students, industry collaborators and policy makers, by answering their questions. The aforementioned users have different abilities in navigating through an interface. Nevertheless, AcademIS interface and visualizations enable the users to find the information that they are seeking, irrespective of their abilities and the user group in which they belong.

TABLE III.

USAGE SCENARIOS AND PATHWAYS Of THE ACADEMIS SySTEM

\section{Education}

S1. A future student wants to select an educational program.

$\mathrm{P} 1$. The student uses the multidimensional comparative ranking, selects the academic departments to be ranked, specifies the weight of each criterion, and examines the ranking results. Then the user selects the curriculum map visualization to overview the curriculum of the academic institution.

S2. A former student needs to inspect the competencies that have been gained after attending the curriculum.

P2. The student uses the AcademIS interface and selects the competency visualization to inspect the acquired competencies.

S3. A current student needs to examine the graduate curricula that the institution offers in order to decide which he/she will attend.

P3. The student accesses the graduate degrees from the AcademIS interface, then selects the scientific areas of interest and inspects the outputs in the interface. 
S4. A professor wants to update his/her academic profile, with the addition of a new publication and a new course.

P4. The professor login to the AcademIS information system and navigates to his/her personal profile. Then the professor adds the necessary information about the course and uploads a .json file about his/her new publication.

\section{Research}

S5. An academic wants to find a researcher to collaborate.

P5. The academic uses the parallel coordinator visualization of I.RE.MA. and inspects which researcher excel, then accesses the web profile of the selected researcher through the AcademIS interface and examines the research activities of the researcher to ensure that they have common research interests.

\section{Cooperation with the industry}

S6. An organization search for academic departments to find interns. P6. The representative of the organization selects the multidimensional comparative ranking component and the academic departments related to the domain of interest, then sorts the results based on a single criterion and decides the academic departments to which the organization will refer to so as to find interns.

$$
\text { Local involvement }
$$

S7. An academic department needs to find another academic department to create a shared curriculum.

P7. The representative of the academic department selects the multidimensional comparative ranking component and the educational criteria of interest and assigns weights in each of them, then chooses with which one to cooperate and selects the academic institution fingerprint to further inspect the educational profile of the selected academic department.

$$
\text { Internationalization }
$$

S8. A researcher wants to find foreign organizations to collaborate in a research project.

P8. The researcher employs the multidimensional comparative ranking and selects research keywords and domains to sort the affiliated organizations of the academic institution, then assigns weights to the criteria, sorts the results based on a single criterion and selects the research collaborator that meet the requirements.

\section{Policy making}

S9. A decision maker that needs to make deductions about the quality of offered services.

P9. The decision maker choose the academic institution fingerprint component and then select the academic department to be evaluated along with a predefined profile for research and inspect the results.

S10. A professor wants to inspect the evaluation results conducted by the students.

P10. The professor accesses the AcademIS interface and selects the profile of the course, then examines the overview of course's evaluation from the students.

In order to present how the system aids the involved stakeholders' in the decision making process, a scenario will be described thoroughly. To be more precise, we will further explore the use case, in which the involved user is a decision maker that needs to make deductions about the ranking of a faculty's academic departments of a specific HEI. The user accesses the AcademIS interface and selects the DS component, then chooses all the academic departments of a faculty of the HEI that will be evaluated by the MCDM procedure. The system assesses the selected departments and the results of the MCDM process are displayed via the parallel coordinates visualization. The parallel coordinates visualization assigns different colors based on the values of a specific dimension, which allows the users to distinguish which records range in the preferable value space for this dimension and to trace these records to the rest axes. In this example, the records are colored based on the axis that represents education. The user decide on the desired value space in the axes of interest, which in this case are education and research and then inspects the records that suffice these criteria. Without the visual analytics component, this scenario would be much more time consuming, it would require much more effort from the users and it would exclude non-informed user groups.

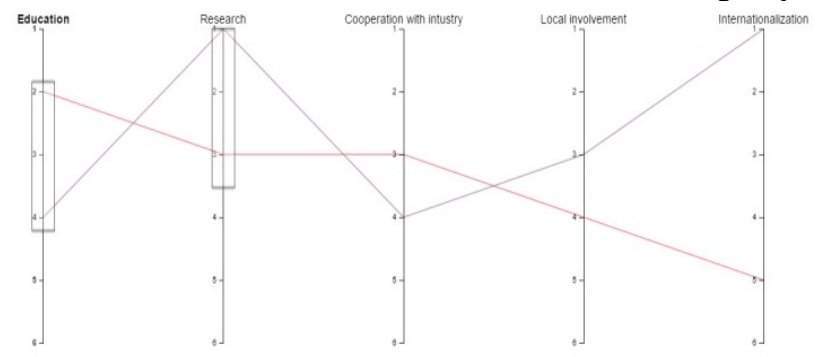

Figure 6. Parallel Coordinates

\section{CONCLUSION}

The AcademIS approach builds upon the semantic technologies to i) increase the availability of academic data by converting them to LOD, ii) make the academic information available using efficient visual interfaces and iii) create the background for their further exploitation by the MCDM component. It offers a dynamic solution to the complex problem of university ranking that ensures the transparency and the reproducibility of the data due to the introduction of Linked Open Data.

It employs the benefits of ontologies to structure the university information and the criteria that measure the university's success on its missions. Not only does it exploit institutional records, but also published Linked Data, accumulating all the existing information about a specific academic unit. It merges the available records with the LODification method, which creates uniformity among the data and enables the user to process all the needed information for an unbiased, multifaceted assessment of educational institutions. AcademIS also propose a visual-aided ontology-based MCDM method for the ranking of HEIs.

Compared to other ranking methods, our approach is reproducible, shareable, extensible and re-usable, due to the use of Linked Open Data. Moreover, because of the visual representation of the data, the information and the ranking results can be easily conceived by any user. The information related to academia can be imported in other systems due to the semantic organization of data. Additionally, by alternating the ontology and applying minor modifications in the information system and the visual MCDM, this methodology can be adapted to any domain, provided that this domain is multidimensional and that the criteria are compatible with those in our approach.

\section{REFERENCES}

[1] F. A. Van Vught and F. Ziegele, "Multidimensional ranking: The design and development of U-Multirank," in Springer Science \& Business Media, vol. 37, 2012. http://dx.doi.org/10.1007/978-94007-3005-2

[2] The Times Higher World University Rankings, The Times Higher Education Supplement, (2015) Available: https://www.timeshighereducation.co.uk.

[3] R. V. Florian, "Irreproducibility of the results of the Shanghai academic ranking of world universities", in Scientometrics, vol. 72 , no. 1,2007 , pp. $25-32$. 
[4] "World Declaration On Higher Education For The Twenty-First Century: Vision And Action", (1998, October 9). Available: http://www.unesco.org/education/educprog/wche/declaration eng. htm\#world declaration.

[5] D. Docampo and L. Cram, "On the internal dynamics of the Shanghai ranking", in Scientometrics, vol. 98, no. 2, 2014, pp. 1347-1366.

[6] L. Waltmanet, C. Calero-Medina, J. Kosten, E. Noyons, R. J. Tijssen, N. J. Eck, T. N. Leeuwen, A.F. Raan, M.S. Visser and P. Wouters, "The Leiden Ranking 2011/2012: Data collection, indicators, and interpretation," Journal of the American Society for Information Science and Technology, vol. 63, no. 12, 2012, pp. 2419-2432. http://dx.doi.org/10.1002/asi.22708

[7] M. Beerkens and D. D. Dill, "The CHE university ranking in Germany," in Public Policy for Academic Quality, Springer Netherlands, 2010, pp. 61-82. http://dx.doi.org/10.1007/978-90481-3754-1_4

[8] A. Y. C. Hou, R. Morse and E. S. Yueh-jen, "Is there a gap between students' preference and university presidents' concern over college ranking indicators?: a case study of "College Navigator in Taiwan"," in Higher Education, vol. 64, no. 6, 2012, pp. 767-787. http://dx.doi.org/10.1007/s10734-012-9524-5

[9] C. Dehon, A. McCathie and V. Verardi, "Uncovering Excellence in Academic Rankings: A case study on the Shanghai Ranking," Scientometrics, vol. 83, no. 2, 2010, pp. 515-524. http://dx.doi.org/10.1007/s11192-009-0076-0

[10] P. Korhonen, H. Moskowitz, and J. Wallenius, "Multiple criteria decision support-A review," European Journal of Operational Research, vol. 63, no.3, 1992, pp. 361-375. http://dx.doi.org/10.1016/0377-2217(92)90155-3

[11] J. Figueira, V. Mousseau and B. Roy, "ELECTRE methods," In Multiple criteria decision analysis: State of the art surveys, Springer New York, 2005, pp. 133-153. http://dx.doi.org/10.1007/b100605

[12] J. Hokkanen and P. Salminen, "ELECTRE III and IV decision aids in an environmental problem," Journal of Multi-Criteria Decision Analysis, vol. 6, no. 4, 1997, pp. 215-226. http://dx.doi.org/10.1002/(SICI)10991360(199707)6:4<215::AID-MCDA139>3.0.CO;2-P

[13] S. K. Card, J. D. Mackinlay and B. Shneiderman, "Readings in information visualization: using vision to think," Morgan Kaufmann, 1999.

[14] B. Ferster, "Interactive visualization: Insight through inquiry," The MIT Press, 2012.

[15] J. J. Thomas and K. Cook, "A visual analytics agenda," Computer Graphics and Applications, IEEE, vol. 26, no. 1, 2006, pp. 10-13. http://dx.doi.org/10.1109/MCG.2006.5

[16] H. Hauser, F. Ledermann, and H. Doleisch, "Angular brushing of extended parallel coordinates," in Information Visualization, INFOVIS 2002, IEEE Symposium, IEEE, 2002, pp. 127-130. http://dx.doi.org/10.1109/infvis.2002.1173157

[17] L. L. Haak, D. Baker, D. K. Ginther, G. J. Gordon, M. A. Probus, N. Kannankutty and B. A. Weinberg, "Standards and infrastructure for innovation data exchange," Science (New York, $N Y$ ), vol. 338, no. 6104, 2012, pp. 196. http://dx.doi.org/10.1126/science. 1221840

[18] K. E. Graham, H. L. Chorzempa, P. A. Valentine and J. Magnan, "Evaluating health research impact: Development and implementation of the Alberta Innovates-Health Solutions impact framework," Research Evaluation, vol. 21, no. 5, 2012, pp. 354367. http://dx.doi.org/10.1093/reseval/rvs027

[19] A. Asserson, K. Jeffery and A. Lopatenko, "CERIF: past, present and future: an overview," in Proceedings of the 6th International Conference on Current Research Information Systems, University of Kassel, Aug. 2002, pp. 33-40.

[20] B. Jörg, "CERIF: The common European research information format model," in Data Science Journal, vol. 9, 2010, pp. 24-31. http://dx.doi.org/10.2481/dsj.CRIS4

[21] J. Bourdeau, R. Mizoguchi, Y. Hayashi, V. Psyche and R. Nkambou, "When the Domain of the Ontology is Education", in Proc. of I2LOR'07, 2007.
[22] C. Ullrich, "Description of an instructional ontology and its application in web services for education," in Proceedings of Workshop on Applications of Semantic Web Technologies for Elearning, $S W-E L$, vol. 4, Nov. 2004, pp. 17-23.

[23] M. Grandbastien, F. Azouaou, C. Desmoulins, R. Faerber, D. Leclet and C. Quénu-Joiron, "Sharing an ontology in Education: Lessons learnt from the OURAL project," in Advanced Learning Technologies, ICALT 2007. Seventh IEEE International Conference, IEEE, July 2007, pp. 694-698. http://dx.doi.org/10.1109/icalt.2007.227

[24] R. Styles, N. Shabir and J. Tennison, "A pattern for domain specific editing interfaces using embedded RDFa and HTML manipulation tools," in Proc. of Scripting and Development for the Semantic Web Workshop at the ESWC, Heraklion, Greece, May 2009, pp. 1613-0073.

[25] D. B. Krafft, N. A. Cappadona, B. Caruso, J. Corson-Rikert, M. Devare, B. J. Lowe and VIVO Collaboration, "Vivo: Enabling national networking of scientists", 2010.

[26] L. Zemmouchi-Ghomari and A. R. Ghomari, "Process of Building Reference Ontology for Higher Education," in Proceedings of the World Congress on Engineering, vol. 3, 2013.

[27] Y. Guo, Z. Pan and J. Heflin, "LUBM: A benchmark for OWL knowledge base systems," in Web Semantics: Science, Services and Agents on the World Wide Web, vol. 3, no. 2, 2005, pp. 158182. http://dx.doi.org/10.1016/j.websem.2005.06.005

[28] K. Möller, M. Hausenblas, R. Cyganiak and S. Handschuh, "Learning from linked open data usage: Patterns \& metrics", 2010.

[29] M. Schmachtenberg, C. Bizer and H. Paulheim, "State of the LOD Cloud 2014", 2014, [Online]. Available: http://linkeddatacatalog.dws.informatik.uni-mannheim.de/state/, [Accessed: 13 March 2015].

[30] P. Jain, P. Hitzler, A. P. Sheth, K. Verma and P. Z. Yeh, "Ontology alignment for linked open data," in The Semantic WebISWC 2010, Springer Berlin Heidelberg, 2010, pp. 402-417. http://dx.doi.org/10.1007/978-3-642-17746-0 26

[31] S. Auer, S. Dietzold, J. Lehmann, S. Hellmann and D. Aumueller, "Triplify: light-weight linked data publication from relational databases", in Proceedings of the 18th international conference on World wide web, ACM, April 2009, pp. 621-630. http://dx.doi.org/10.1145/1526709.1526793

[32] T. Berners-Lee, "Linked data-design issues," 2006, [Online]. Available: http://www.w3.org/DesignIssues/LinkedData.html, [Accessed: 13 March 2015].

[33] C. Bizer, R. Cyganiak and T. Heath, "How to publish linked data on the web," in Tutorial in the 7th International Semantic Web Conference, Karlsruhe, Germany, Oct. 2007.

[34] M. Saeki, and H. Kaiya, "On relationships among models, meta models and ontologies," in Proceedings of the Proceedings of the 6th OOPSLA Workshop on Domain-Specific Modeling (DSM 2006), Oct. 2006.

[35] S. Bechhofer, I. Buchan, D. De Roure, P. Missier, J. Ainsworth, J. Bhagat, P. Couch, D. Cruickshank, M. Delderfield, I. Dunlop, M. Gamble, D. Michaelides, S. Owen, D. Newman, S. Sufi and C. Goble, "Why linked data is not enough for scientists", in Future Generation Computer Systems, vol. 29, no. 2, 2013, pp. 599-611. http://dx.doi.org/10.1016/j.future.2011.08.004

[36] M. Schmachtenberg, C. Bizer, A. Jentzsch and R. Cyganiak, "Linking Open Data cloud diagram 2014", [Online]. Available: http://lod-cloud.net/, [Accessed: 13 March 2015].

[37] A. Tsolakidis, C. Sgouropoulou, I. Xydas, O. Terraz and G. Miaoulis, "Academic research policy-making and evaluation using graph visualization", in Informatics (PCI), 2011 15th Panhellenic Conference on, IEEE, Sept. 2011, pp. 28-32.

[38] C. Sgouropoulou, E. Triperina and A. Tsolakidis, "Fostering academic collaboration for quality education and research: the AcademIS case," EDULEARN14 Proceedings, 2014, pp. 16-26.

[39] J. Hebeler, M. Fisher, R. Blace and A. Perez-Lopez, "Semantic web programming", John Wiley \& Sons, 2011. 


\section{AUTHORS}

E. Triperina is with the XLIM laboratory of the University of Limoges, CNRS, UMR 7252, 123 avenue Albert Thomas Limoges, Limousin 87000, France and with the Department of Informatics of the Technological Educational Institute of Athens Ag. Spyridonos St., 122 10, Egaleo, Greece (e-mail: evatrip@teiath.gr).

C. Sgouropoulou is with the Department of Informatics of the Technological Educational Institute of Athens Ag. Spyridonos St., 122 10, Egaleo, Greece (email: csgouro@teiath.gr).

I. Xydas is with the Department of Informatics of the Technological Educational Institute of Athens Ag.
Spyridonos St., 122 10, Egaleo, Greece (e-mail: yxydas@teiath.gr).

O. Terraz is with the XLIM laboratory of the University of Limoges, CNRS, UMR 7252, 123 avenue Albert Thomas Limoges, Limousin 87000, France (e-mail: terraz@unilim.fr).

G. Miaoulis is with the Department of Informatics of the Technological Educational Institute of Athens Ag. Spyridonos St., 122 10, Egaleo, Greece (e-mail: gmiaoul@teiath.gr) and with the XLIM laboratory of the University of Limoges, CNRS, UMR 7252, 123 avenue Albert Thomas Limoges, Limousin 87000, France.

Submitted, 09 September 2015. Published as resubmitted by the authors on 10 October 2015. 